\title{
A new dynamic visual illusion: The bending hourglass
}

\author{
JAMES T. WALKER \\ University of Missouri, St. Louis, Missouri 63121
}

\begin{abstract}
A white hourglass moving laterally across a black surface appears to bend in such a way that the narrow portion lags behind its true position, but only when a stationary point is fixated. Visually tracking the moving hourglass eliminates the apparent distortion. However, visually tracking an object moving in front of a stationary white hourglass produces the illusory bending, although saccadic eye movements across the hourglass do not. A black hourglass, moving or stationary, presented against a white background produces no illusory bending. These effects are explained in terms of the differential visual persistence of the wide and narrow portions of the hourglass figure.
\end{abstract}

Consider a slender white hourglass figure 4 to 6 in. high, similar in shape to the middle drawing in Figure 1. Now suppose such an hourglass moves left and right across a black surface while the observer fixates a stationary point on that surface. Depending on the velocity of movement, the white hourglass appears bent approximately as in $1 \mathrm{~L}$ or $2 \mathrm{~L}$ (see Figure 1 ) as it moves to the left, and bent as in $1 R$ or $2 R$ as it moves to the right. In addition, the white hourglass appears to widen as it moves, especially the narrow middle portion, which appears to trail behind its true position. Moving a white hourglass by hand over a distance of a foot or so, at about 1 oscillation/sec, readily produces the illusory bending, as long as the observer fixates a stationary point. When the observer visually tracks the moving hourglass, the distortion is eliminated.

Now consider a stationary white hourglass lying on a black surface. When a moving object-the point of a pencil, for example-moving from left to right in front of the stationary hourglass is visually tracked, illusory bending, as in $1 \mathrm{~L}$ or $2 \mathrm{~L}$, is produced. Here, the sense of motion of the stationary hourglass across the retina is opposite to that of the moving object, and the direction of the illusory bending depends on the retinal motion of the white hourglass. Saccadic eye movements across the stationary hourglass, between fixation points to the left and to the right of the figure, produce no illusory bending.

A black hourglass moving across a white surface produces no illusory bending, whether a stationary point is fixated or the moving figure is tracked. Visually tracking an object moving in front of a stationary black hourglass also produces no apparent distortion, and neither do saccadic eye movements across a stationary black hourglass.

A version of this paper was presented at the annual meeting of the Psychonomic Society in Philadelphia, Pennsylvania, November 13,1981 .

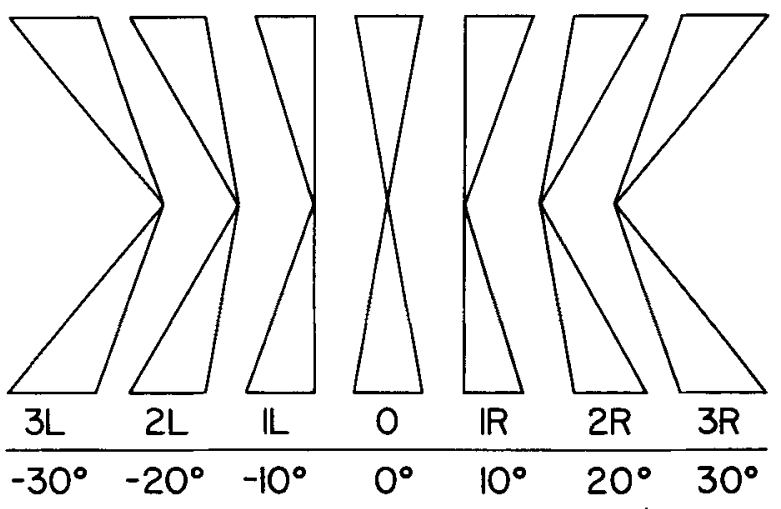

Figure 1. Drawings used in Experiment 2 (reduced in size). Subjects used scale values from $3 L$ to $3 R$ to indicate perceived bending. Degrees of bending, shown here, were not shown to the subjects.

The illusory bending of a moving or stationary white hourglass depends on the differential visual persistence of the wider and narrower portions of the figure. Visual persistence, the length of time a stimulus remains visible after its offset, is inversely related to the duration of the stimulus (Bowen, Pola, \& Matin, 1974; Coltheart, 1980; Efron, 1970; Haber \& Standing, 1970). Thus as the image of a white hourglass sweeps across the retina, the duration of the narrower portion of the figure is shorter than that of the wider portions, and thus the narrower portion appears to trail farther behind its true position because of its greater visual persistence. The absence of distortion in a black hourglass can also be accounted for in terms of visual persistence, as explained in the discussion section. Indeed, the illusory appearance of the white hourglass and the absence of distortion in the black hourglass were derived a priori from considerations of visual persistence, and the present observations and experiments were carried out to test those derivations. 


\section{EXPERIMENT 1}

This experiment utilized white and black hourglasses moving back and forth across horizontal surfaces in the left-right and up-and-down directions.

\section{Method}

Subjects. Seven men and three women, mostly faculty and graduate students in the Department of Psychology at the University of Missouri-St. Louis, participated as subjects.

Materials. The black and white hourglasses were $15.2 \mathrm{~cm}$ high, and the sides made an angle of $25 \mathrm{deg}$ with each other. The narrow portion of each hourglass was $3 \mathrm{~mm}$ wide. The white hourglass was moved across a large black horizontal surface, and a sheet of $8 / 12 \times 11 \mathrm{in}$. $(21.6 \times 27.9 \mathrm{~cm})$ white paper served as a background for the black hourglass. The luminance of the white hourglass and the white paper background ranged from about 20 to $80 \mathrm{~mL}$.

Procedure. All subjects viewed both hourglasses, half viewing the black one, and the other half the white one, first. Each hourglass was presented lying flat on a horizontal surface. The hourglass was oriented vertically, in the sense that its long axis was parallel to the direction of the sides of a page. The experimenter moved the hourglass back and forth across the surface in the horizontal dimension by a finger placed near one end of the hourglass. The rate of movement was about 1 oscillation/ sec. The subjects were instructed to fixate a point near the center of the black or white surface as steadily as possible and were asked to describe the appearance of each hourglass.

\section{Results}

All 10 subjects reported illusory bending of the white hourglass in the expected direction. The middle of the hourglass appeared to lag behind its true position, and to point away from the direction of motion (binomial $\mathrm{p}<.002$, two-tailed). No subject reported any illusory bending of the black hourglass $(\mathrm{p}<.002)$.

After viewing the vertically oriented black and white hourglasses, eight of the subjects were shown the white hourglass with its long axis oriented horizontally, mov. ing up and down at the same rate as before. Those subjects were asked to compare the magnitude of apparent distortion produced by the vertical and horizontal motion of the hourglass. Seven subjects reported greater illusory bending in the horizontally moving figure, and one subject reported no difference between the effects of horizontal and vertical motion. Those results were statistically significant by a sign test $(p<.02$, twotailed).

\section{EXPERIMENT 2}

This experiment was designed to measure the apparent bending of the hourglass under better controlled conditions than the previous experiment.

\section{Method}

Subjects. Ten men and 10 women, students in an undergraduate psychology course in research methods, participated as subjects.

Apparatus. A rotating drum, $15 \mathrm{~cm}$ in diameter and $17 \mathrm{~cm}$ in height, carried the visual displays. The axis of the drum was vertical. The drum was mounted on a table top a little below eye level at a viewing distance of $120 \mathrm{~cm}$ from the subject. The sides of the black and white hourglasses made angles of $20 \mathrm{deg}$ with each other, and the hourglasses were $12.7 \mathrm{~cm}$ in height, subtending visual angles of about $6 \mathrm{deg}$. The narrow portion of each hourglass was $3 \mathrm{~mm}$ wide. The black hourglass was presented against a white background, and the white hourglass against a black background. A projector lighted the visual displays, providing a luminance of $10 \mathrm{~mL}$ for the white hourglass and for the white background against which the black hourglass was presented, and a luminance of $.28 \mathrm{~mL}$ for the black hourglass and the black background. A black spot, $6 \mathrm{~mm}$ in diameter, was projected onto the center of the rotating drum to serve as a stationary fixation point. The spot was clearly visible against the black as well as the white background. An electric motor rotated the drum clockwise or counterclockwise, providing motion from right to left or left to right as seen by the subject. The drum rotated at $60 \mathrm{rpm}$, yielding a linear velocity of about $22 \mathrm{deg}$ of visual angle per second at the center of the display.

Procedures. All subjects were shown the black and white hourglasses moving in both directions, left and right. Half the subjects viewed the black hourglass first, and half viewed the white one first. The sexes were balanced across those viewing conditions. The same sequence of directions (left-right or rightleft) was used for any particular subject for both hourglasses. Those sequences were randomized across subjects, with the restriction that half the subjects be run using each sequence.

Each subject was instructed to fixate the black dot as steadily as possible, and to indicate which of the numbered drawings in Figure 1 appeared most similar to the moving hourglass. A copy of those drawings was attached to the table top directly in front of the subject and was illuminated by the stray light from the projector. Each drawing was the same height as the hourglass on the rotating drum. The subjects were instructed to use fractional numbers between the integer scale values if they wished, and seven subjects made use of such fractional scale values.

\section{Results}

The data were subjected to a three-way analysis of variance in which the factors were sequence of colors (black-white, white-black), color of hourglass (black, white), and direction of motion (left, right). There were repeated measures on the last two factors. The sequence of colors was incorporated as a factor since it was thought that viewing the white hourglass first-seeing its obvious bending-might predispose those subjects to look more carefully for bending in the black hourglass. However, there was no significant main effect of sequence $[F(1,18)=2.36, p>.10]$, no significant sequence $X$ color interaction or sequence $X$ direction interaction (neither $F>1$ ), and no sequence $X$ color $X$ direction interaction $[F(1,18)=2.01, p>.10]$. There was no significant main effect of color $(F<1)$, but there was a main effect of direction $[F(1,18)=$ $43.96, p<.001]$, and a significant color $X$ direction interaction $[F(1,18)=15.49, p<.001]$, all as expected.

Figure 2 shows the results graphically. The black hourglass produced no illusory bending for either direction of motion, but the white hourglass appeared bent in one direction as it moved to the left and bent in the opposite direction as it moved to the right-hence the color $X$ direction interaction, and the main effect of direction.

\section{GENERAL DISCUSSION}

The shapes of the hourglasses used in the present experiments were chosen on the basis of pilot observations. 


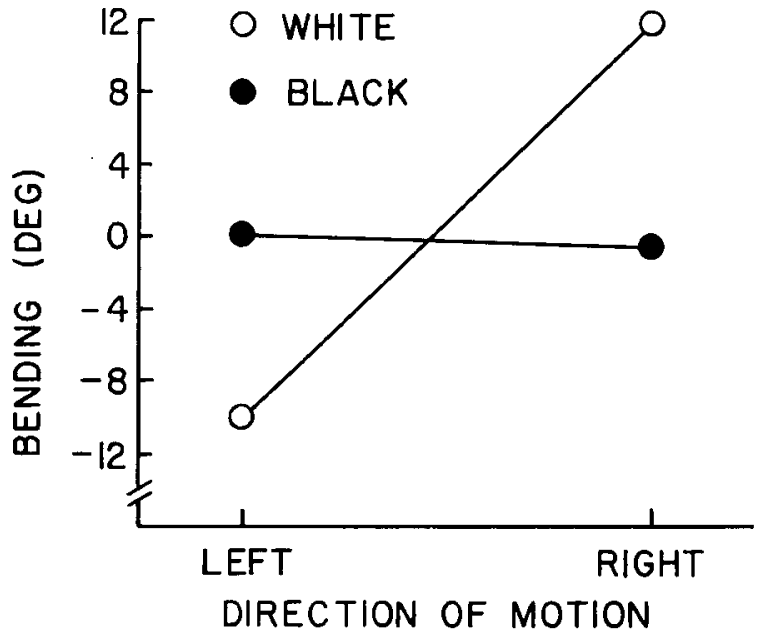

Figure 2. Mean illusory bending in Experiment 2 by color of hourglass and direction of motion. Degrees of bending are illustrated in Figure 1.

A very slender white hourglass, whose sides make an angle of $5 \mathrm{deg}$, shows little illusory bending-and a slender rectangle, whose sides are parallel, shows none. A white hourglass whose sides are at right angles undergoes great apparent distortion, in that the narrow portion lags far behind its true position. But the distortion in such an hourglass produces less apparent bending, and appears more difficult to assess quantitatively, than the distortions observed in more slender hourglasses.

As noted earlier, previous research has shown that visual persistence is inversely related to the duration of a visual stimulus (see Coltheart, 1980, for an excellent review of the persistence literature). In the case of the moving white hourglass, as the observer fixates a stationary point, the narrow portion of the hourglass illuminates retinal points very briefly while the wider portions illuminate other retinal points for longer periods of time. Thus, because of its greater persistence, produced by its shorter duration, the narrow portion appears to widen in relation to the remainder of the moving image and appears to lag behind its true position, thereby producing the illusory bending of the hourglass.

Visually tracking an object moving from left to right in front of the stationary white hourglass produces the same kind of illusory bending that motion of the hourglass from right to left would produce. The image of the stationary hourglass sweeps across the retina in a direction opposite to that of the moving object, and it is the retinal motion that produces the illusory bending through the mechanism described above. The failure of saccadic eye movements to produce any apparent distortion may represent a variety of saccadic suppression (Matin, 1974) or may be due to the high velocity of saccadic movements.

In the case of the black hourglass moving against a white background, the background illuminates all points in the retinal image except for the brief periods of time when the illumination is turned off by the passage of the black hourglass. If the black hourglass sweeps across the field of view once per second, then all points in the retinal image are illuminated for only a little less than a second-regardless of whether the illumination at a particular point is turned off by the narrow or wider portion of the hourglass. Thus, all points in the retinal image are lighted for nearly the same relatively long period, nearly $1 \mathrm{sec}$, and thus there should be little or no differential visual persistence between the wider and narrower portions of the black hourglass. Indeed, if there were any such noticeable differential persistence, then the black hourglass should appear to bend in the direction opposite to the illusory bending in the white hourglass.

The above explanation of the absence of illusory bending in the black hourglass ignores the possibility that a black stimulus-as well as a white one- might also remain visible after its termination, perhaps through the mediation of "off" cells (Kuffler \& Nicholls, 1976). If the visual persistence of black were to obey the same laws as the persistence of white, then it would seem that the black and white hourglasses should undergo similar illusory distortions. However, the roles of lightness and darkness are clearly not symmetrical in some perceptual situations. For example, it is very difficult to recognize a familiar face in a photographic negative, even though the negative contains the same visual information as a positive print. The results of the present experiments offer further evidence of the lack of symmetry between the roles of lightness and darkness in determining visual form perception.

Visual persistence also accounts for spatial distortions in other situations. For example, a strobe flash on a moving airplane appears to lag behind its true position if the observer fixates a stationary point near the flight path (Walker \& Irion, Note 1); and Morgan (1977) has offered an explanation of the Pulfrich effect based on differential visual persistence produced by filtering one eye. Those earlier studies, and the present results, indicate that visual persistence, besides merely extending the perceived duration of a stimulus, may strongly influence the perception of form and space when motion is involved.

\section{REFERENCE NOTE}

1. Walker, J. T., \& Irion, A. L. Apparent displacement of moving and stationary strobe flashes. Paper presented at the annual meeting of the Psychonomic Society, St. Louis, Missouri, November 13, 1980.

\section{REFERENCES}

Bowen, R. W., Pola, J., \& Matin, L. Visual persistence: Effects of flash luminance, duration, and energy. Vision Research, $1974,14,295-303$. 
Coltheant, M. Iconic memory and visible persistence. Perception \& Psychophysics, 1980, 27, 183-228.

EFron, R. Effect of stimulus duration on perceptual onset and offset latency. Perception \& Psychophysics, 1970, 8, 231-234.

Haber, R. N., \& Standing, L. G. Direct estimates of the apparent duration of a flash. Canadian Journal of Psychology, $1970,24,216-229$.

Kuffter, S. W., \& Nicholls, J. G. From neuron to brain: $A$ cellular approach to the function of the nervous system. Sunderland, Mass: Sinauer, 1976.
Matin, E. Saccadic suppression: A review and an analysis. Psychological Bulletin, 1974, 81, 899-917.

Morgan, M. J. Differential visual persistence between the two eyes: A model for the Fertsch-Pulfrich effect. Journal of Experimental Psychology: Human Perception and Performance, 1977, 3, 484-495.

(Manuscript received May 26, 1981; accepted for publication August 5, 1981.) 